\title{
Extraction of Silica Nanoparticles from Rice Husk Ash (RHA) and Study of Its Application in Making Composites
}

\author{
Bimal Kumar Raut, Khim Prasad Panthi* \\ Department of Chemistry, Tri-Chandra Multiple Campus, Tribhuvan University, Kathmandu, Nepal \\ *Corresponding E-mail: khimcdc@gmail.com \\ (Received: Sept. 30, 2019; Revised: Dec. 24, 2019 \& Accepted: Dec. 29, 2019)
}

\begin{abstract}
Nanotechnology is the most emerging field in the area of different scientific research. Various methods of synthesis of nanoparticles are available. The wet chemical synthesis method is applicable in the extraction of silica nanoparticles from Rice Husk Ash (RHA). Rice husk is a form of waste product from the rice milling industries which is produced in an abundance amount in and around the country. Rice husk which is generally left on the field as a waste contains $60 \%$ silica content and can be economically viable raw materials for the extraction of silica [1-2]. Initially, Rice husk was burnt to obtain its ash. Then, a simple bottom-up approach, the sol-gel method was applied, and fine powder silica in the nanoscopic range was extracted. After extraction of it, internal arrangement and average particle size were recognized by XRD while molecular components and structure present in silica were identified by FTIR. The obtained silica was then used in making of ceramics matrix nanocomposites (particularly silica-sand cement block), and its mechanical properties were identified by compressive strength test using Instron testing machine which was found to be increased in comparing with the compressive strength of ceramics composite (Sand cement block) prepared in the same ratio and same size of mold as that of ceramics matrix nanocomposites. The ceramics matrix nanocomposites acquiring higher mechanical strength than Ceramics composite occurred due to the incorporation of Silica nanoparticles.
\end{abstract}

Keywords: Rice husk, sol-gel method, ceramics matrix nanocomposites, ceramics, composite, silica nanoparticles

\section{Introduction}

The project work was carried out with an objective to extract silica nanoparticles from rice husk ash (RHA), the silica content in rice husk ash and to prepare composite using extracted silica nanoparticles to find out whether nanoparticles affect in mechanical properties of composite or not. Nanoparticles are nanomaterials having all three dimensions in the range of 1-100 nm [3]. All nanoparticles are nanomaterials but not all nanomaterials are nanoparticles. Nanomaterials are materials having at least one of their dimensions in the range of 1-100 $\mathrm{nm}$. This range is called nanoscale.

Rice is the second most widely consumed food item globally, with rice paddy production registering about 758 million metric tons in 2070 , a number that will increase gradually due to the projected demand of the world population [4]. The RHA is an agricultural waste, which is generally used as ameliorants to break up clay soils and improved soil structure but is also used for the production of silica. The properties of RHA depend on the ecological circumstances of its origin as well as the process applied for burning the husk [5]. Silica which is the constituents of silicon and oxygen has the molecular formula $\mathrm{SiO}_{2}$. Silica has been widely used in vegetable oil refining, pharmaceutical products, detergents, adhesive, chromatograph column planning and ceramics [6-8]. Not only this, silica is also found to be used in water purification, vulcanizing rubber, control of insect pests, in stored food stuffs, manufacturing refractory bricks, and as admixture in low cost concrete block manufacturing [9].

Abundantly available waste RHA has been widely used as raw materials for the preparation of silica gel 
and powder following (Kamath and Protocor, 1998) [1].

Nanoparticles exhibit attractive properties such as low thermal expansion, high mp, high thermal stability, high thermal resistance and chemical stability $[10,11]$. The interfacial area that creates a significant volume fraction of the interfacial polymer with the properties different from bulk polymer even at low filter loadings $[12,13]$. Several researchers have studied the effect of particle size and volume fraction on the mechanical response of composite [14-18]. However, among the numerous polymer composites; silica polymer nanocomposites are most commonly reported in the literature and are also employed in variety of applications, such as electronics, automotive and aerospace industries as well as used in many industrial products due to their good mechanical characteristics [19].

Ceramics is defined as the solid materials that are not metal, plastic, or derived from plants or animals [20]. Ceramics have some distinctive properties including good chemical inertness, high temperature stability brittleness, high $\mathrm{mp}$ and an electrical insulation capability [21]. Furthermore, Chemical bonding (both covalent and non covalent) between filler and a polymer improves materials compatibly, and thus enhances certain properties of matrix above and beyond what is accomplished by single doping with filler [22-24]. Using similarly sized dopant and matrix elementary building blocks may have certain distinctive advantages [25].

\section{Materials and Methods}

Chemicals used: Distilled water, $1 \mathrm{~N} \mathrm{NaOH}, 1 \mathrm{~N} \mathrm{HCl}$

Apparatus used: Beaker, conical flask, burner, wire gauze, magnetic stirrer, filter paper (Whatmann 41), hot air oven

\section{Preparation of chemical reagents}

\section{Preparation of $1 \mathrm{~N} \mathrm{NaOH}$}

$$
\begin{gathered}
\text { Mass of } \mathrm{NaOH} \text { required }(\mathrm{w})=\mathrm{NEV} / 1000 \\
\text { Where, } \mathrm{N}=\text { Normality, } \mathrm{E}=\text { Equivalent wt., } \\
\qquad \mathrm{V}=\text { Volume required in } \mathrm{mL}
\end{gathered}
$$

For $600 \mathrm{~mL}$, mass of $1 \mathrm{~N} \mathrm{NaOH}$ required was $24 \mathrm{~g}$. It was taken in a volumetric flask and little amount of distilled water was added to dissolve it. After it got fully dissolved, distilled water was added upto the mark of $600 \mathrm{~mL}$.

\section{Preparation of $1 \mathrm{~N} \mathrm{HCl}$}

As concentrated $\mathrm{HCl}$ has approximately strength of $36 \%$, its normality calculated to be $11.6 \mathrm{~N}$ by using the following formula:

$$
\begin{aligned}
& \text { Where, } \% \text { by mass }=36 \\
& \text { Specific gravity }=1.1789 \\
& \text { Equivalent weight }=36.5
\end{aligned}
$$$$
\text { Normality }=\frac{(\% \text { by mass } \mathrm{x} \text { specific gravity } \mathrm{x} 10)}{\text { Equivalent weight }}
$$

For $200 \mathrm{~mL}$ of $1 \mathrm{~N}$ dilute $\mathrm{HCl}, 17.24 \mathrm{~mL}$ of concentrated $\mathrm{HCl}$ was added up to $200 \mathrm{~mL}$ of distilled water. It was calculated by the following way:

$$
\text { (Conc. HCl) } \mathrm{V}_{1} \mathrm{~N}_{1}=\mathrm{V}_{2} \mathrm{~N}_{2} \text { (1 N Dil. Hcl) }
$$

Where, $\mathrm{V}_{1}=$ Volume of concentrated $\mathrm{HCl}$ to be calculated

$\mathrm{N}_{1}=$ Normality of Concentrated $\mathrm{HCl}(11.6 \mathrm{~N})$

$\mathrm{V}_{2}=$ Volume of dilute $\mathrm{HCl}$ to be prepared $(200 \mathrm{~mL})$

$$
\mathrm{N}_{2}=\text { Normality of dilute } \mathrm{HCl}(1 \mathrm{~N})
$$

\section{Preparation of rice husk ash from rice husk}

Rice Husk was collected from the nearest Rice milling industry, which was then burnt and converted into ash. The ash converted was then made into fine powder.

\section{Extraction of silica from rice husk ash}

This was done by Sol-gel method. In this method, $50 \mathrm{~g}$ RHA was weighed and dispersed in $300 \mathrm{~mL}$ of distilled water in a beaker. The $\mathrm{pH}$ of solution maintained at 7 by testing with litmus paper. The solution was then stirred by magnetic stirrer for 2 hours.

It was then filtered by Whatmann 41 filter paper and the residue was washed with $500 \mathrm{~mL}$ of water.

The residue was then dispersed in $300 \mathrm{~mL} 1 \mathrm{~N} \mathrm{NaOH}$ and boiled with constant stirring for 1 hour. It was then filtered through whatmann 41 filter paper. The residue was then washed with $500 \mathrm{~mL}$ boiling water. The filtrate was collected and allowed to cool. Then, the filtrate was titrated with $1 \mathrm{~N} \mathrm{HCl}$ till the $\mathrm{pH}$ of filtrate became neutral.

When $\mathrm{pH}$ of filtrate became 7, gel obtained. The gel was allowed to age for 18 hours. After 18 hours, 500 $\mathrm{mL}$ of distilled water was added into volumetric flask containing gel and crushed the gel. After crushing the 
gel, the solution was left for some time, later which two layers were seen. Upper layer comprised of water called supernatant and lower layers consisted of gel.

The supernatant was discarded by the help of dropper and the washing process was repeated for 3 more times. The gel obtained after removing supernatant was kept in an oven for 18 hours at 80 degree Celsius. The gel produced called xerogels, which was then kept in air tight plastic bottle.

The method applied following (Kamath and Protocor, 1998) [1] can be explained step wise as:

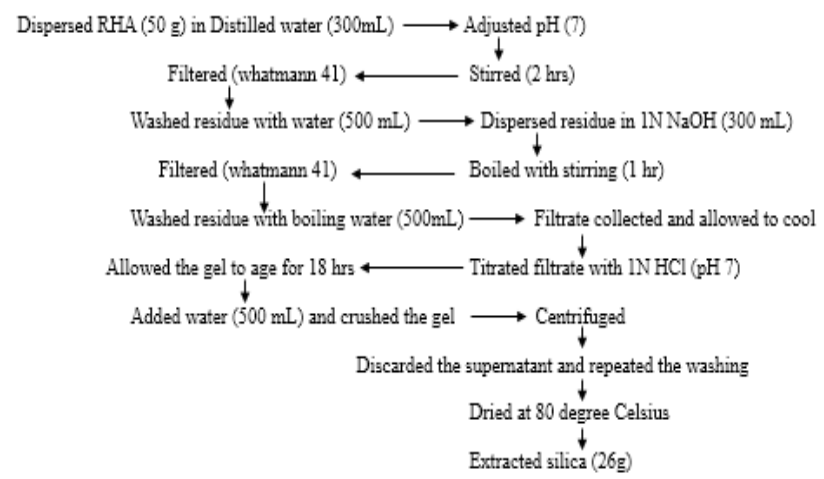

\section{Preparation of ceramics matrix nanocomposites (CMNs)}

The CMNs, silica-sand cement block was prepared. For this purpose, the extracted silica from RHA was mixed to the sand cement mixture, which is believed to increase the mechanical property. Firstly extracted silica, sand and cement were mixed in the porcelain basin in the ratio of 1:4:1. Then, distilled water (generally $26-33 \%$ of the total weight of mixtures) was added on it till the required texture was obtained. After then, it was framed in a $2 \mathrm{~cm}$ cube mold and the curing process was done by immersing the block in distilled water. As 3 days strength test was followed, the blocks were removed from distilled water after low-cost days and allowed it to dry, free from any moisture. The compressive test of the block was then done.

\section{Ceramics composites}

The preparation method was the same as that of CMNs, silica-sand cement block. The only difference is that silica nanoparticles were not added to cement and sand.

In this process, sand and cement were taken in the porcelain basin in the ratio of 4:1 and mixed properly. Then distilled water was added till the required texture was obtained. After then, framing was done in $2 \mathrm{~cm}$ cube mold. Like the preparation of the ceramics matrix nanocomposite block, the curing process and strength testing procedure of the ceramics composite block was done by the same method.

The main purpose behind the preparation of ceramics composite was to find the difference between mechanical strength of ceramics matrix nanocomposite block containing nanoparticles (silicasand cement block) and the ceramics composite block without nanoparticles (sand cement block).

\section{Results and Discussion}

\section{FTIR analysis}

The FTIR of silica nanoparticles shows various peaks as a result of the absorption of light of a wavelength in infra red region. From the graph of Transmittance\% vs. wave number per $\mathrm{cm}$, it is cleared that rocking vibration occurs at $800 \mathrm{~cm}-1$. Similarly; Si-O asymmetrical stretching vibration occurs at $1068 \mathrm{~cm}^{-1}$.

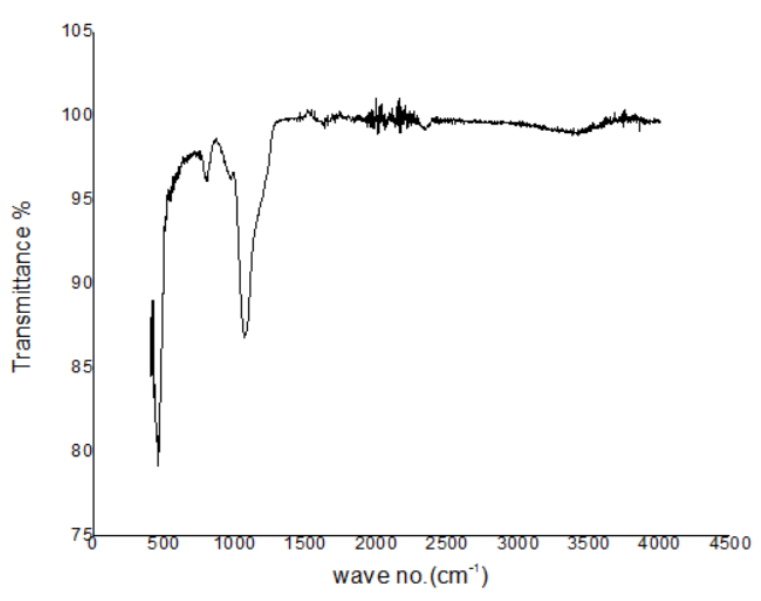

Figure 1: FTIR of silica nanoparticles

Some very small peaks are due to instrumental error but chemists found it as a result of carbon dioxide.

Table 1: FTIR data sample containing silica nanoparticles

\begin{tabular}{|l|l|l|}
\hline S. N. & Wave number $\mathbf{c m}^{-1}$ & Description \\
\hline 1. & 800 & Rocking vibration \\
\hline 2. & 1068 & Si-O-Si asymmetrical stretching vibration \\
\hline
\end{tabular}




\section{XRD analysis}

Confirmation of the effectiveness and the size of silica nanoparticles were provided by X-ray studies (XRD). The center of peak and the value of FWHM required to calculate the size of nanoparticles was determined by using origin software.

On the basis of X-ray curves, Lorentzian fitting was done. It is intended to a one-mode system and believes to describe a decaying system over time. The data of XRD (two theta degree value vs. intensity) was copied into origin software. Then, two strong peaks were selected efficiently and analyzed in multi-peaks fitting. As mentioned above, Lorentzian fitting was done which then by double-clicking on the center of peaks gave the value of angle of the center of peak

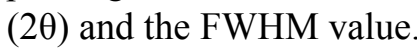

The center of peak and FWHM value on using origin software found to be 32.10910 and 0.37580 . The value of $\theta$ calculated by dividing the center of the peak by 2 and FWHM value was then converted into radian which found to be $6.55 \times 10-3$. The wavelength used was $1.5406 \AA$ and Scheerer's constant value $(\kappa)$ used was 0.94 . Thus, by using Scheerer's formula, the size of the particle was determined.

$$
\mathrm{D}=\mathrm{K} \lambda / \beta \operatorname{Cos} \theta
$$

Thus, putting all the values, the particle size was found to be $23 \mathrm{~nm}$.

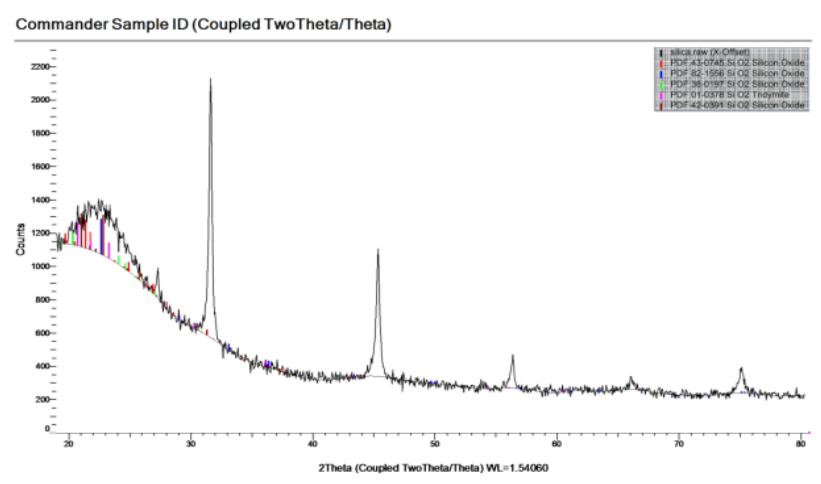

Figure 2: $X R D$ of silica nanoparticles

\section{Compressive test}

At first, the cross-sectional area and the breaking load of both the blocks, ceramics matrix nanocomposite (containing silica nanoparticles), and another ceramics composite (without silica nanoparticles) were measured and their value was found to be:

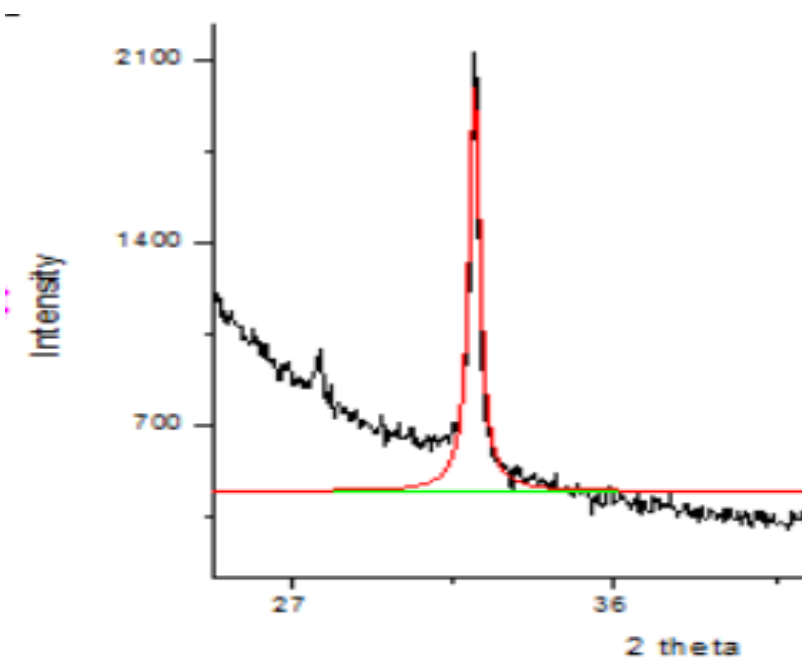

Figure 3: Lorentzian fitting for prominent peak of silica nanoparticles

Table 2: Cross-sectional area and breaking load of blocks

\begin{tabular}{|c|c|c|}
\hline Block & $\begin{array}{c}\text { Cross-sectional } \\
\text { Area(cm }\end{array}$ & Breaking load(N) \\
\hline $\begin{array}{c}\text { Ceramics Matrix } \\
\text { Nanocomposite block }\end{array}$ & 4 & 40 \\
\hline $\begin{array}{c}\text { Ceramics Composite } \\
\text { block }\end{array}$ & 4 & 24 \\
\hline
\end{tabular}

Thus, by putting the value in a formula,

$$
\text { Compressive strength }=\frac{\text { Breaking load }}{\text { Cross-sectional area }}
$$

It was found that the compressive strength of block containing silica nanoparticles (ceramics matrix nanocomposite) was $10 \mathrm{~N} / \mathrm{cm}^{2}$ and $6 \mathrm{~N} / \mathrm{cm}^{2}$ for the block having no silica (ceramics composite).

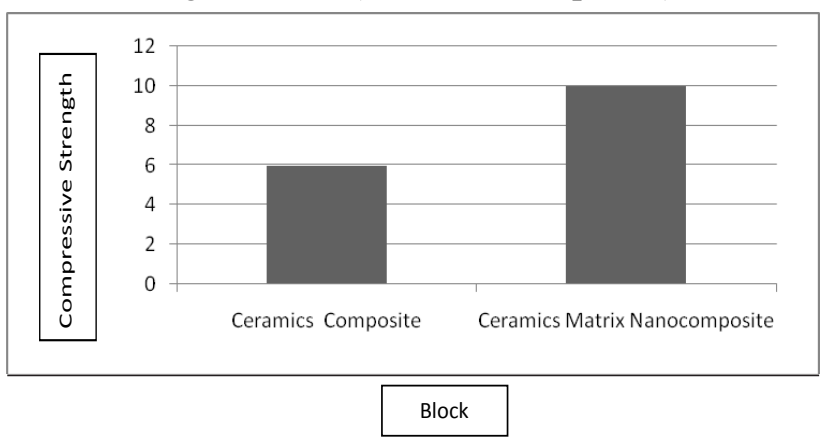

Figure 4: Bar Diagram showing the result of compressive test 


\section{Conclusion}

Silica nanoparticles were synthesized from Rice Husk Ash (RHA) by using the sol-gel method. The synthesized silica nanoparticles found to be $26 \mathrm{~g}$ $(52 \%)$, which is almost equal to $60 \%$ and seems to follow the citation [1,2]. The Silica nanoparticles were characterized by spectroscopic techniques like FTIR and XRD. As per the result of XRD, the particle size was found to be $23 \mathrm{~nm}$. By analyzing the data of FTIR, rocking vibration, and Si-O-Si asymmetrical stretching vibration was found. Similarly, synthesized silica nanoparticles were then incorporated in sand cement mixture in 1:4:1 ratio to prepare ceramics matrix nanocomposite block. In the same way, ceramics composite block was also prepared where silica nanoprticles were not incorporated in the sandcement mixture. The mechanical property of prepared blocks was characterized by compressive strength test using the Instron testing machine. As per the result of compressive test, the ceramics matrix nanocomposite block containing the silica nanoparticles found to have almost 1.67 times greater strength than the ceramics composite block containing no silica. This proves that silica nanoparticles enhances the mechanical strength and hence it can be used in several construction materials.

\section{Acknowledgements}

I would like to thank my friend Dipa Aryal, Krishna G. C. and Hemraj Upadhyay. I am very thankful to Dr. Suresh Dhungel, Senior Scientist at NAST, Assoc. Prof. Dr. Surendra Kumar Gautam, Department of Chemistry, Tri-Chandra Multiple Campus and Asst. Prof. Achyut Adhikari, Central Department of Chemistry, Tribhuvan University. This work was carried out at the lab of Tri-Chandra Multiple Campus, Ghantaghar, Kathmandu. XRD was carried out at NAST, Satdobato. FTIR was carried out in the Central Department of Chemistry, Kirtipur. The mechanical property was tested in the Soil Lab of Pulchowk Engineering Campus. I am thankful to all of them.

\section{References}

1. S. R. Kamath and A. Proctor, Silica gel from rice husk ash: preparation and characterization, Cereal Chemistry, 1998, 75, 484-487.

2. A. Chakraverty and S. Kalemullah, Conversion of rice husk into amorphous silica and combustible gas, Energy Consers. Mgmt., 1991, 32, 565-570.

3. S. K. Murthy, Nanoparticles in modern medicine: State of the art and future challenges, International Journal of Nanomedicine, 2007, 2(2), 129-141.

4. K. S. Lokare, Rising from the ashes: renewable silica from rice husk ash, Advanced BioFuels USA, 2017.

5. M. R. F. Gonzlaves and C. P. Bergmann, Thermal insulators made with rice husk ashes: production and correlation between properties and microstructure, Constr. Build Materials, 2007, 21(12), 2059-2065.

6. C. J. Brinker and G. W. Scherer, Applications. in: sol-gel science, the physics and chemistry of sol-gel processing, Academic Press, San Diego, CA, 1990, 839-880.

7. R. K. Iler, Silica gels and powders, The Chemistry of Silica, 1979, 462-599.

8. A. Proctor, P. K. Clark and C. A. Parker, Rice hull ash adsorbent performance under commercial soy soil bleaching conditions, Journal of the American Oil Chemists' Society, 1995, 72, 459462.

9. U. Kalapathy, A. Proctor and J. Shultz, A simple method for production of pure silica from rice hull ash, Bioresource Technology, 2000, 73, 257 262.

10. W. K. Kriven, J. F. Siah, M. Schmucker et al., High temperature microhardness of single crystal, Journal of the American Ceramic Society, 2004, 87(5), 970-972.

11. R. R. Tumalla, Development of high performance interfill material for system chip technology, Journal of the American Ceramic Society, 1991, 74(2), 895-899.

12. A. C. Balazas, T. Emrick and T. P. Russel, Nanoparticle polymer composites, Where two small world meet, Science, 2006, 314, 11071110 .

13. R. Krishnamoorti and R. A. Vaia, Polymer nanocomposites, Journal of Polymer Science Part B-Polymer Physics, 2007, 45, 3252-3256. 
14. A. C. Moloney, H. H. Kausch and H. R. Stieger, The fracture of particulate filled epoxide-resins. Journal of Material Science, 1984, 19, 11251130.

15. M. Frounchi, T. A. Westgate, R. P. Chaplin and R. P. Burford, Fracture of polymer networks based on diethylene glycol bis(allyl carbonate), Polymer, 1994, 35, 5041-5045.

16. F. Stricker, Y. Thomann and R. Mulhaput, Influence of rubber particle size on mechanical properties of polypropylene-SEBS blends, Journal of Applied Polymer Science, 1998, 68, 1891-1901.

17. R. T. Quazi, S. N. Bhattacharya and E. Kosior, The effect of dispersed paint particles on the mechanical properties of rubber toughened polypropylene composites, Journal of Material Science, 1999, 34, 607-614.

18. A. Boonyapookana, K. Nagata and Y. Mutoh, Fatigue crack growth behavior of silica particulate reinforced epoxy resin composite, Composite Science and Technology, 2011, 71, 1124-1131.
19. A. Moisala, Q. Li, I. A. Kinloch and A. H. Windle, Thermal and electrical conductivity of single and muti-walled carbon nanotube epoxy composites, Composites Science and Technology, 2006, 66, 1285-1288.

20. D. W. Richerson, The Magic of Ceramics, American Ceramic Society, Wiley 2012, $2^{\text {nd }}$ Ed.

21. M. N. Rahaman, Ceramic Processing and Sintering. Marcel Dekker, New York, 2003, $2^{\text {nd }}$ Ed., 1-2.

22. Y. Chen and J. O. Iroh, Chemistry of Materials, 1999, 11, 1218.

23. Z. Ahmad and, J. E. Mark, Chemistry of Materials, 2001, 13, 3320.

24. S. Campbell and D. Scheiman, High Performance Polymers, 2002, 14, 17.

25. C. A. Mitchell, J. L. Bahr, S. Arepalli, J. M. Tour and R. Krishnamoorti, Macromolecules, 2002, 35, 8825 .

26. D. Rolison, Science, 2003, 299, 1698. 